\title{
L'enseignement de la civilisation : évolution et représentations dans le champ de la revue Le Français dans le Monde (1961 -1976)
}

Résumé de la thèse de doctorat soutenue par Evelyne Argaud (juin 2000, directeur de thèse: $G$. Zarate)

\section{Evelyne Argaud}

\section{(2) OpenEdition}

\section{Journals}

Édition électronique

URL : https://journals.openedition.org/dhfles/2064

DOI : $10.4000 /$ dhfles.2064

ISSN : 2221-4038

\section{Éditeur}

Société Internationale pour l'Histoire du Français Langue Étrangère ou Seconde

Édition imprimée

Date de publication : 1 juin 2001

Pagination : 169-184

ISSN : 0992-7654

Référence électronique

Evelyne Argaud, «L'enseignement de la civilisation : évolution et représentations dans le champ de la revue Le Français dans le Monde (1961 -1976) ", Documents pour I'histoire du français langue étrangère ou seconde [En ligne], 26 | 2001, mis en ligne le 04 septembre 2014, consulté le 21 septembre 2021. URL : http://journals.openedition.org/dhfles/2064 ; DOI : https://doi.org/10.4000/dhfles.2064

Ce document a été généré automatiquement le 21 septembre 2021.

(c) SIHFLES 


\section{L'enseignement de la civilisation : évolution et représentations dans le champ de la revue Le Français dans le Monde (1961 -1976)}

Résumé de la thèse de doctorat soutenue par Evelyne Argaud (juin 2000, directeur de thèse: $G$. Zarate)

\section{Evelyne Argaud}

1 Cette recherche, qui souhaite être une contribution à l'histoire de la didactique du français langue étrangère (F.L.E), s'est proposé d'étudier, sur une période de quinze ans, la place, l'évolution et les représentations de l'enseignement de la civilisation française dans la revue pédagogique Le Français dans le monde, fondée par André Reboullet qui en fut le premier rédacteur en chef de 1961 à 1981. C'est dans l'optique d'un regard réflexif d'un champ sur son propre passé que se situe ce travail qui désire s'ajouter aux études déjà entreprises dans l'histoire récente de la didactique du E.L.E, de sa théorie et de ses pratiques ${ }^{1}$.

2 La recherche comprend quatre grandes parties qui correspondent à trois grandes étapes. La première de ces parties est consacrée à la présentation de l'appareil théorique et méthodologique; la seconde construit l'espace objectif de la revue (environnement, création, acteurs divers). Enfin, dans un troisième temps, (3e et $4 \mathrm{e}$ parties), l'ouvrage, en introduisant l'expérience des auteurs/agents à travers l'examen de leurs discours, de leurs références bibliographiques, des mots-clés utilisés, étudie la mise en place et l'évolution d'une pratique professionnelle et d'un espace de réflexion consacré à l'enseignement/étude de la civilisation française dans le cadre du champ du français langue étrangère. La thèse comprend par ailleurs un index des notions, des noms propres et différentes annexes. 


\section{Constitution du corpus (sélection et périodisation)}

3 L'étude d'une revue présente l'avantage d'offrir un corpus déjà constitué; cependant, à l'intérieur de ce corpus, il n'en fallait pas moins procéder à un travail de sélection des articles de civilisation et définir les notions mêmes d'article et celle de civilisation. Sans reprendre les nombreux débats concernant les termes « culture » et « civilisation », la thèse rappelle l'évolution des deux mots, telle qu'elle apparaît dans des dictionnaires du XIX et XXe siècle et dans le champ du F.L.E, ce qui permet de mettre en relief le rapprochement sémantique des deux mots. Sont définies également les différentes approches de la civilisation (littéraire, sociologique, anthropologique, sémiologique) et la façon dont ces approches ont été considérées par les auteurs du champ du F.L.E. La constitution du corpus a conduit à sélectionner dans la revue tous les articles de réflexion théorique sur l'enseignement de la civilisation, les dossiers et études de civilisation qui traitaient de sujets très divers, les fiches pédagogiques et les documents audio-visuels qui abordaient des sujets de civilisation, les articles de la chronique « vie quotidienne " animée par René Pucheu. Ce journaliste, qui possédait également une formation en droit et qui écrivait dans d'autres revues, est resté longtemps responsable de cette rubrique dont une des fonctions était de renforcer l'orientation choisie par la revue. Il s'agissait alors de faire obstacle à une conception traditionnelle de la civilisation et de mettre l'accent sur la civilisation du quotidien, sur la vie de tous les jours dans ce qu'elle peut avoir de plus banal, tout en réhabilitant cette banalité et en en faisant un moyen de connaissance en profondeur d'une société.

Ce travail de sélection a conduit à l'établissement d'un relevé chronologique de tous les articles, qui figure en annexe et où ont été précisés la date de parution de l'article, le nom de l'auteur, sa fonction, ce qui a constitué un outil fondamental de travail. Ce relevé permet de voir rapidement, pour chaque année, combien d'articles ont été publiés, lesquels et par qui. On peut ainsi constater la continuité ou les variations en fonction des années et les mettre éventuellement en rapport avec le contexte social et historique: c'est ainsi que la revue, qui se voulait pourtant un moyen d'information sur la France contemporaine, ne dit rien des événements d'Algérie ni de ceux de mai-juin 68 , si ce n'est de façon indirecte et allusive.

5 L'auteur a choisi de s'intéresser aux quinze premières années d'existence de la revue, à une période où le champ du F.L.E s'institutionnalise sous l'influence d'importants bouleversements historiques: décolonisations, grande expansion économique, période de reconstruction de l'après-guerre et reconsidération de la place de la France dans le monde. La deuxième guerre mondiale et les décolonisations avaient entraîné une redistribution des positions internationales qui, pour la France, s'était traduite par la perte du statut de grande puissance. Le traumatisme causé par la défaite et l'occupation avaient considérablement entamé le prestige de la France qui, de grande puissance, devait se résigner peu à peu à n'être plus qu'une puissance moyenne. L'enseignement de la civilisation, étroitement lié aux représentations que l'on se fait de soi-même et de l'étranger, apparaissait comme un domaine particulièrement sensible aux conséquences d'une nouvelle donne internationale, pour un pays contraint de réajuster ses ambitions et désireux cependant de maintenir son rayonnement par d'autres liens que ceux de la contrainte passée. Cette nouvelle situation internationale devait inévitablement influencer l'image de la civilisation française et engendrer un discours plus modeste dont la présence devait être repérable dans la revue. 
6 Le corpus comprend 125 numéros et prend fin au n 125, en décembre 1976. On peut s'interroger sur le choix de cette clôture; plusieurs raisons sont avancées par la recherche: deux numéros spéciaux (121, mai-juin 76 et 125, nov-déc.76) témoignaient d'un renouveau de l'intérêt pour la sociologie. A.Reboullet souhaitait construire les dossiers sur la notion sociologique de "rôle » empruntée au sociologue Henri Mendras. Par ailleurs, Louis Porcher, docteur d'état en sociologie, était devenu directeur du CREDIF (Centre de recherche et d'étude pour la diffusion du français) et mettait l'accent sur la sociologie afin de rétablir un équilibre face à la linguistique toute-puissante. De plus, le développement à la même époque, dans les années 75-76, de l'approche communicative permettait d'intégrer dans la compétence de communication une composante socioculturelle. Cela ne veut pas dire que la sociologie ait été ignorée auparavant, loin de là, ni que l'approche de la civilisation ait été profondément modifiée dans la revue à partir du $\mathrm{n}^{\circ} 125$. Il y a eu toutefois un essoufflement, une certaine lassitude d'une approche didactique fondée essentiellement sur le lien languecivilisation, qu'accompagnait un désir de renouveler l'orientation des dossiers. Ces éléments ont semblé suffisants pour clore un corpus, dont la durée correspondait à une période qui avait vu l'essor d'un discours dominant sur l'enseignement de la civilisation, discours porté essentiellement par une institution le BEL/BELC (Bureau d'étude et de liaison pour l'enseignement du français dans le monde) qui, d'ailleurs, réduit à partir de ce moment-là, tout au moins de façon provisoire, le nombre de ses dossiers.

7 Très vite, le besoin s'est fait sentir d'établir une périodisation pour ces quinze ans et d'opérer un découpage dans la période retenue. Cette périodisation a été mise en place à partir des changements observés dans la revue: titres des articles, disparitions ou entrée en scène d'auteurs-phares, thèmes et textes choisis, orientations générales. $\mathrm{A}$ été ainsi délimitée une première période qui va du n 1 à 46 (mai 61 à février 67) et qui est marquée par un débat entre les notions de culture et de civilisation, par la nécessité pour la civilisation de prendre ses distances avec une conception qui l'assimilerait à la " culture cultivée ", par la volonté de se libérer de l'influence de la littérature pour se tourner vers une culture de la vie quotidienne. Ce débat a été intitulé par la recherche " Entre tradition et modernité » et durant cette période, on constate un relatif équilibre des forces en présence. La revue se cherchait une ligne pédagogique dans le domaine de l'enseignement de la civilisation, ce dont témoigne le $\mathrm{n}^{\circ} 16$ (avril-mai 63) et plus tard le $\mathrm{n}^{\circ} 37$ (décembre 65) consacré à la civilisation du quotidien. Un tel débat peut sembler peu original et banal, tant toute époque est toujours, d'une façon ou d'une autre, transition; cependant il se révélait inévitable, d'autant plus qu'il va s'affaiblir et perdre de son intérêt dans la 2e période. Cette 2e période, qui commence au n ${ }^{\circ} 47$ (février 47) pour prendre fin au n ${ }^{\circ} 125$ (décembre 76), a été intitulée «la civilisation sous tutelle de la langue ». L'enseignement de la civilisation, libéré de la tutelle de la littérature, va être, sous l'impulsion du BELC et de Francis Debyser, directeur du BELC à partir de 1967, étroitement associé à celui de la langue, lui-même fortement déterminé par les recherches en linguistique, ce qui toutefois n'élimine pas les approches sociologique et anthropologique. Cependant, selon F. Debyser, ces approches sont moins efficaces que la linguistique pour donner une cohérence et une unité à l'enseignement de la civilisation qui reste une discipline très éclectique.

8 Le corpus, ainsi délimité, a été enrichi par les entretiens que l'auteur a pu avoir avec certains acteurs et collaborateurs de la revue, entretiens menés à partir de quelques 
questions précises ou plus larges et qui ont apporté des informations complémentaires. Parmi les personnes interrogées figuraient André Reboullet, Guy Michaud auteur du Guide France et fondateur de la revue Tendances, René Pucheu, Jacques Pécheur (ancien rédacteur en chef de la revue), Jean Cayol, collaborateur de Guy Michaud à la revue Tendances et auteur d'études et de dossiers de civilisation dans Le Français dans le Monde. Cependant, ces entretiens ne sauraient être intégrés au corpus car ils n'ont pas été assez nombreux pour cela. Enregistrés, ils ont généralement été très ouverts et s'assimilaient à des conversations libres, à partir d'un canevas très souple. Il ne s'agissait nullement de les transcrire ensuite intégralement ni de construire des catégories d'analyse mais de solliciter des témoignages de la part d'acteurs qui avaient joué un rôle fondamental ou important dans la revue.

\section{Appareil méthodologique et théorique}

9 La recherche s'inscrit dans une démarche pluridisciplinaire qui emprunte à l'histoire, la sociologie et la linguistique. L'approche pluridisciplinaire est apparue indispensable, non seulement en raison de la nature du domaine étudié dont on a souligné plus haut la diversité, mais aussi parce que la civilisation est une discipline "à très haute teneur idéologique $»^{2}$, pour laquelle un choix pédagogique est porteur d'un certain nombre de données culturelles. Discipline idéologique, l'enseignement de la civilisation ne pouvait pas faire l'économie d'une articulation avec l'environnement historique, la politique culturelle française et la question de la place que la France tenait à occuper dans le monde. La revue, en tant que phénomène culturel, s'insérait inévitablement dans un contexte historique dont il fallait tenir compte. Cependant, on ne saurait se dissimuler les difficultés de cette approche pluridisciplinaire, d'autant qu'il n'existe, du moins à notre connaissance, aucune théorie qui articule histoire, sociologie et linguistique; la perspective retenue offrait, avec ses insuffisances, l'occasion d'utiliser les apports de différentes disciplines.

\subsection{La perspective historique}

Afin de situer la revue dans son contexte historique, la recherche a eu recours à des ouvrages qui traitaient de la politique culturelle française et de l'histoire de la diplomatie française postérieure à la 2e guerre mondiale. La civilisation touchant largement au domaine des idées, l'ouvrage de Raoul Girardet L'idée coloniale en France, outre qu'il abordait une période qui concernait directement le domaine étudié, s'intéressait aux mentalités collectives et analysait, en distinguant des familles d'esprit, les représentations du fait colonial dans les esprits. L'enseignement de la civilisation, marqué par une tradition missionnaire, ne pouvait rester étranger aux débats analysés par R. Girardet d'autant plus que la revue est apparue en pleine guerre d'Algérie ${ }^{3}$ et juste après les décolonisations de 1960. L'une des hypothèses de départ de la recherche était que les débats provoqués par ces bouleversements avaient dû inspirer indirectement, mais de façon déterminante, la réflexion didactique sur l'enseignement de la civilisation; il s'agissait de savoir comment la revue avait considéré et traité l'héritage colonial, comment l'idéologique s'inscrivait éventuellement dans le pédagogique et si les catégories mises en place par R.Girardet pouvaient être utiles. Le passé était présent dans les pages de la revue sous la forme des termes de l'ancien discours colonial (cause, mission, civilisation, universel, rayonnement) et la réflexion sur 
l'enseignement de la civilisation devait conserver les traces d'une lutte entre deux états du champ, entre des attitudes et des comportements différents.

\subsection{Le recours à l'analyse de discours}

11 Toute activité de recherche suppose une tentative de classification et bien que cette activité soit délicate, car on peut toujours hésiter entre l'observation de ce qui est repérable et généralisable et l'affirmation de la différence, de l'irréductibilité des textes, il n'en était pas moins nécessaire de trier cette vaste masse d'articles et de distinguer certains types de discours dans la revue. Les articles n'avaient pas tous la même fonction dans la revue: la recherche menée a ainsi constaté la présence de trois types de discours concernant la civilisation, qui ont été identifiés à la suite du comptage des occurrences du mot « civilisation » et ces discours recoupent en partie les catégories d'articles mises en place par la revue. Toutefois cette correspondance n'est pas systématique et à un type de discours ne répond pas forcément une catégorie d'auteurs même si on peut observer certaines régularités. La thèse n'a pas souhaité s'appesantir sur des débats théoriques et s'est intéressée, dans le cadre de sa perspective pluridisciplinaire, à la définition que donne R.Robin de l'analyse de discours : « objet frontière » qui « travaille aux limites des grands découpages disciplinaires "4; à cette définition, elle en ajouté une autre qu'elle a jugée opératoire pour son projet: c'est celle que propose D.Maingueneau lorsqu'il prend le mot dans sa plus large extension et qu'il entend par discours toute production écrite ou orale, qui suppose un locuteur et un auditeur en interaction, définition qui s'inspire de Benveniste et qui convenait tout à fait à un corpus d'articles écrits pour un public précis, et dans lequel, « explicite ou non la relation de personne est présente partout $»^{5}$.

La thèse relève ainsi un premier discours sur la civilisation française elle-même, qui relève du domaine sociologique ou anthropologique, où le terme « civilisation » désigne une collectivité humaine précise ou plus générale, dans l'espace ou dans le temps; ce discours correspondait à une diffusion de savoirs socioculturels dans des domaines très variés (études), réalisées souvent par des universitaires de disciplines variées, mais pas exclusivement, sur des sujets très divers, composées d'un texte entièrement rédigé par leurs auteurs qui s'adressent à des non-spécialistes et qui relèvent du discours de vulgarisation. Un deuxième discours, essentiellement pédagogique ou méthodologique, se rencontre fréquemment dans les dossiers pédagogiques ou dans les articles relevant d'une réflexion didactique et/ou méthodologique sur l'enseignement de la civilisation. Ce discours s'interroge sur le concept de civilisation, sur les documents à utiliser, sur le contenu à donner au cours de civilisation, sur les rapports entre langue et civilisation. Il est souvent pris en charge par des spécialistes de français langue étrangère qui peuvent avoir ou non un statut universitaire et qui sont parfois des formateurs de formateurs. Les discours de ces spécialistes empruntent leurs références à des théories diverses et ils les reformulent pour un public non-spécialisé d'enseignants; il s'agit là d'un discours intermédiaire, de vulgarisation, sans que ce mot ait ici la connotation péjorative qu'on lui donne parfois, un discours de retransmission et de reformulation de savoirs théoriques appliqués à l'enseignement de la civilisation. Un troisième discours, enfin, peu fréquent, tenu en général par des responsables, des "décideurs ", s'interrogeait sur la place de la France dans le monde, sur la politique culturelle et linguistique de celle-ci. Son examen permettait d'inscrire les deux premiers dans un cadre plus général, celui de la politique culturelle de la France à l'étranger. L'ignorer 
serait revenu à ignorer des éléments importants des " conditions de production " des discours de la revue sur l'enseignement de la civilisation. Ces trois discours n'étaient pas étanches d'où la difficulté à les analyser séparément, cependant cette classification, pour imparfaite qu'elle soit, constituait un point de départ qui pouvait aider, à la fois, à sérier les problèmes et à répondre à des questions initiales posées par la recherche: quelle représentation le premier discours construisait-il de la civilisation française ? Quels étaient les différents objectifs du discours didactique de vulgarisation ? Était-ce un discours qui permettait de rendre le professeur plus compétent, de s'affirmer dans un champ en voie de formation et d'acquérir une reconnaissance, qui cherchait à modifier des représentations et des pratiques?

Pour répondre à ces questions, la thèse a cherché à savoir sur quelles références bibliographiques ${ }^{6}$ se fondaient les articles de civilisation de la revue, références qui se manifestaient aussi bien dans les œuvres et les noms d'auteurs cités que dans les citations directes ou indirectes. Pour cela, a été fait un répertoriage des domaines évoqués à travers les termes qui les désignaient; ce répertoriage posait des problèmes spécifiques en raison de la polysémie des termes et de la difficulté à les sélectionner : fallait-il ainsi regrouper les adjectifs correspondant aux formes substantivées et fallaitil, par exemple, relever, entre autres, social et sociologie, littéraire et littérature ? Ce relevé, avec ses limites et ses insuffisances, permettait de voir quelles disciplines avaient joué un rôle important et lesquelles avaient été sollicitées pour modifier les pratiques pédagogiques. C'est ainsi que la linguistique, très peu sollicitée durant la première période distinguée, fait une entrée en force impressionnante durant la seconde période, contribuant un peu plus au discrédit de l'ancienne approche littéraire. Cependant, le nombre d'occurrences n'était pas un critère à lui seul suffisant. Certaines occurrences pouvaient se concentrer dans certains articles et chez certains auteurs. Le grand nombre de citations ne signifie pas une attitude homogène à l'égard d'un domaine cité. Ainsi, la littérature souvent mentionnée, l'était fréquemment pour être critiquée comme mode d'approche de la civilisation, ce qui témoignait du rôle considérable qu'elle avait joué et de sa situation de monopole durant une très longue période.

14 La thèse $a$, de même, pour chaque article, relevé ses références nominatives, quand il y en avait, et dressé un tableau des spécialistes cités au moins deux fois, ce qui a permis de voir qui citait qui, de noter les changements d'une période à l'autre et d'observer quelles étaient les références les plus fréquentes. Barthes est ainsi l'auteur le plus cité dans la revue pour cette période. L'auteur de Mythologies et de Rhétorique de l'image a véritablement constitué une référence pour l'analyse de ce que l'on a appelé les « faits de civilisation ». D'une façon générale, l'examen des références bibliographiques signale une méfiance à l'égard de la tradition humaniste classique, une ligne générale que l'on peut qualifier de moderne et de progressiste, mais avec mesure, qui refuse un modernisme intolérant, soucieuse de ne pas donner prise au reproche de soumission à la mode et qui ne répugne pas à citer les " anciens ", à condition qu'ils ne présentent pas de traits conservateurs accusés.

L'étude des citations et de la manière dont les auteurs les employaient et les inséraient dans leurs articles était elle aussi intéressante. En utilisant les catégories exploitées par D.Maingueneau ${ }^{7}$, on constate que les auteurs de la revue font souvent appel à la citationpreuve afin de soutenir une argumentation, une idée, et à laquelle on a recours parce que l'auteur est une figure d'autorité. Les citations ne sont pas utilisées pour être 
discutées ou mises en regard avec d'autres mais pour étayer, le plus souvent, une démonstration ou une position. Les références rendent ainsi possible la constitution d'un discours et d'un domaine qui peut s'auto-identifier par rapport à d'autres, ce qui explique que les références aux spécialistes hors-champ soient nettement plus nombreuses que les références internes au champ.

\subsection{La perspective sociologique}

La recherche a essayé d'articuler positions et discours en s'intéressant à la place occupée par les acteurs/auteurs de la revue et en ayant recours à la notion de champ ${ }^{8}$, le champ étant un microcosme relativement indépendant où agissent des acteurs, microcosme qui a des enjeux propres mais qui entretient des relations avec d'autres champs proches. La notion de champ est une construction scientifique qui n'est jamais définitive, qui entretient des rapports étroits avec la réalité mais qui n'est pas cette réalité. Il ne s'agissait pas de faire une étude sociologique de la revue en raison de la difficulté et de l'impossibilité matérielle à réunir certaines données et parce qu'il est très délicat $d$ 'objectiver un champ auquel on appartient. De plus, une telle étude aurait conduit à s'éloigner des discours tenus sur renseignement de la civilisation. Cependant il était possible de classer, en tenant compte de toutes les difficultés liées à l'opération de classement, les auteurs du champ de la revue et plus particulièrement ceux qui avaient écrit sur l'enseignement de la civilisation, en utilisant certains critères comme la nationalité, la fonction, l'espace professionnel (enseignants du secondaire, du supérieur, didacticiens, politiques, administratifs), la matière enseignée, le lieu géographique de travail, l'appartenance ou non au champ du F.L.E; on pouvait ainsi déterminer pour ces auteurs, du moins pour certains d'entre eux, leur capital à partir des indications données par la revue ou par des informations recueillies ailleurs, dans d'autres revues, ou grâce encore aux entretiens, ce qui permettait d'établir une relation entre la place occupée dans le champ et les discours tenus. C'est ainsi que les auteurs de la revue sont représentés globalement par deux grandes catégories: les universitaires et ceux qui appartiennent à une institution F.L.E, comme le BELC qui est très représenté dans la revue. Ces deux catégories n'interviennent pas de la même façon. Les universitaires écrivent ponctuellement, apportent leur savoir, parfois leur notoriété (capital symbolique) mais ne s'investissent pas durablement dans la revue; les spécialistes du BELC, institution récente et fragile, en marge de l'université, interviennent de façon beaucoup plus régulière avec un investissement professionnel beaucoup plus fort, et ce sont eux qui, sous l'influence de l'évolution des sciences humaines, ont contribué à moderniser l'enseignement de la civilisation en mettant en place un nouveau discours dominant. Ils se sont opposés ainsi aux représentants d'une conception plus traditionnelle de la civilisation, représentants peu nombreux dans la revue et dont l'une des caractéristiques principales était d'avoir suivi une formation initiale littéraire et d'appartenir parfois à L'Alliance française, institution plus ancienne et reconnue depuis longtemps.

La recherche a fait appel à une autre notion sociologique qui est celle d'« habitus ", inséparable de celle de champ puisqu'elle permet de rendre compte des comportements des agents sociaux. Bourdieu la définit comme un ensemble de " principes de vision et de division ${ }^{9}$ qui correspondent à des goûts, des préférences, des opinions qui déterminent des pratiques et que les agents ne mettent pas toujours en relation avec le groupe social auquel ils appartiennent. Ils génèrent des pratiques et des 
représentations "qui peuvent être objectivement adaptées à leur but sans supposer la visée consciente de fins. ${ }^{10}$ On retrouve ici la notion de représentation et sans vouloir neutraliser les différences, on ne peut manquer de constater que le sociologue H.Mendras pose également qu'il existe des « attitudes », dotées d'une certaine stabilité mais capables bien sûr d'évoluer, attitudes faites d'opinions, d'idées, de préférences et/ ou de rejets qui s'expriment avec une certaine cohérence. C'est cette cohérence qui fait que la connaissance approfondie des opinions d'un groupe ou d'une personne dans une situation donnée permet de déduire ou de prévoir ses réactions ou ses opinions dans une autre circonstance. On voit que cette notion d'« attitudes » peut recouper certains aspects de celle d'« habitus ». Selon Bourdieu, l'habitus est tout entier traversé par l'histoire, tout en conservant une certaine autonomie par rapport à celle-ci. Ainsi, des agents peuvent conserver un habitus ancien et présenter des comportements qui ne sont plus adaptés à de nouvelles conditions historiques; cette notion de décalage sera opératoire pour le débat tradition/modernité qui a traversé la revue; certains des représentants ou partisans de la traditionnelle approche littéraire de la civilisation ressentaient avec amertume leur déphasage et la dépréciation de leurs valeurs (en particulier toutes les représentations qui donnaient de la France l'image d'un pays de culture et d'art, porteur d'une civilisation universelle et chargé d'une mission) et ils avaient nettement le sentiment d'être dépassés, d'autant plus que le prestige de la France à l'étranger s'était fondé sur ces anciennes valeurs, ce qui engendrait chez certains le sentiment de vivre une période de décadence et d'y assister réduits à l'impuissance.

Par ailleurs, un agent social a l'habitus de son champ qu'il n'a pas forcément choisi. Son comportement ne s'explique donc pas par une volonté consciemment calculatrice mais il est le résultat de dispositions acquises socialement et de l'illusio, autre concept que Bourdieu préfère à celui d'intérêt car il est moins connoté moralement. L'illusio c'est le désir socialement constitué d'un agent par son habitus de s'investir dans des enjeux propres au champ dont il relève, au-delà d'une adhésion librement consentie ou d'une pure contrainte mécanique due au milieu. Il existe ainsi des conduites objectivement adaptées à une fin précise et qui sont vécues par les agents sociaux comme totalement désintéressées. Cette notion permet de rendre compte des distorsions entre les comportements objectifs des agents sociaux et le vécu subjectif qu'ils ont de leurs comportements. C'est ainsi que l'on a noté la contradiction que recelait autrefois une présence coloniale assurée sur le terrain par un corps enseignant parfois hostile au colonialisme ou tout au moins plus sensible que d'autres secteurs à l'influence de l'anticolonialisme. Nous avons relevé également la contradiction entre l'image de neutralité pédagogique que la revue a tenu à donner d'elle-même à plusieurs reprises et une présence politique dans ses pages qui se manifestait de différentes façons aussi bien par des interventions officielles que par des silences ou certains discours militants. La revue a rempli une des missions qui étaient les siennes, à savoir moderniser les pratiques pédagogiques, afin de remplacer la contrainte passée propre à l'époque coloniale par une coopération qui tienne compte des nouvelles donnes historiques et qui restaure une primauté intellectuelle française à l'étranger. Se présenter comme un outil purement pédagogique au service des professeurs de français escamotait la fonction politique au sens large de la revue et cette conviction qu'avaient les acteurs/ collaborateurs de la revue de remplir une fonction purement pédagogique était absolument nécessaire à la réalisation de la fonction politique. Cela dit, il est très difficile de mesurer l'efficacité réelle de la revue sur la modification des pratiques de 
l'enseignement et des représentations de la civilisation française, et là n'était pas l'objet de la thèse.

\subsection{La notion de représentation}

19 La notion de représentation est elle-même au croisement de plusieurs champs, social, culturel, linguistique, psychologique. C.Kerbrat-Orecchioni note que l'idéologie entretient avec "la compétence linguistique des relations aussi étroites qu 'obscures ${ }^{11}$ les sociologues, comme Pierre Bourdieu en particulier, ont montré que la représentation d'un objet fait partie de sa réalité. La représentation que l'on a de sa propre civilisation détermine nécessairement les pratiques que l'on met en place pour l'enseigner et cette représentation est d'autant plus forte qu'elle s'impose comme une évidence au sujet qui ignore qu'elle est une construction historique et relative. La notion de représentation présente ainsi l'intérêt de reconnaître le rôle du sujet social dans l'élaboration d'un savoir sur la réalité sociale. Or, dès que l'on aborde les problèmes posés par la notion de représentation, on s'affronte également à une série d'autres notions dont les contenus sont parfois très proches : valeurs, croyances, opinions, attitudes, idées, mythes, symboles, normes; ces notions, qui forment une constellation, relèvent toutes de l'activité mentale ou psychique. Pour un sociologue comme Henri Mendras, mythe et idéologie sont à la fois des termes synonymes et des instruments d'analyse, la sémiologie nous a appris aussi que toute société engendre ses propres mythes; le mythe moderne est ainsi un système logique intellectuel qui permet de rendre compte de certaines réalités, de leur donner une interprétation et une cohérence. Pour certains auteurs de la revue, il y a ainsi un « mythe » de la civilisation française solidement installé dans les esprits depuis très longtemps et qu'il faut remettre en question.

Le travail entrepris désirait repérer les traces des représentations anciennes dans les articles de la revue, identifier les auteurs qui soit les reprenaient en y adhérant, soit les remettaient en question, examiner si de nouvelles valeurs tentaient de détrôner les anciennes et si celles-ci devenaient à leur tour des normes. La revue avait forcément joué un rôle fondamental dans cette entreprise puisque toute activité intellectuelle crée des symboles, des représentations. Une des hypothèses de la recherche était que la présence de ces valeurs anciennes devaient se manifester à travers quelques notions fondamentales repérables dans des mots-clés qu'il convenait de sélectionner et d'étudier dans les articles de la revue. Ces termes, tels que universel, rayonnement, cause, nation, mission, vocation, ont été choisis parce que leur présence signalait un discours traditionnel, discours qui a été celui de la France par le passé, et qu'ils permettaient également d'aborder le débat signalé plus haut entre tradition et modernité. La recherche s'est intéressée ainsi à l'entourage contextuel des mots-clés, la façon dont les auteurs les considéraient, entre adhésion ou distance. L'examen de l'attitude du sujet parlant à l'égard de son énoncé s'est faite à l'aide des notions propres à l'analyse de discours, telles que celle de "distance ". La distance, beaucoup plus nette que l'adhésion, présentait toutes les nuances : de la méfiance indécise au rejet pur et simple des mots-clés observés. Ont été étudiés également les marqueurs de subjectivité, en particulier tous les termes qui signalent un rapport personnel à un objet («notre, nos, nous, notre pays, nos amis étrangers, nos écrivains ») et qui témoignaient, chez les auteurs d'articles, d'un engagement émotionnel ou au contraire d'une prise de distance à l'égard de la société ou du fait de civilisation qu'ils étaient chargés de présenter à leurs lecteurs. 
21 L'énonciation, en s'intéressant à la subjectivité dans le langage, rejoint ainsi les préoccupations de la sociologie. Mendras note que les "attitudes sont généralement polarisées, chargées d'affectivité sur un sujet donné, (...) il y a toujours une attitude pour et une attitude contre $»{ }^{12}$ L'approche énonciative et l'analyse du discours, en intégrant le lexique, l'étude du positionnement de la personne, la modalisation ou la marque donnée par le sujet à son énoncé, la place occupée par les destinataires, à travers la façon dont ils sont désignés explicitement ou implicitement, permettaient de compléter les points de vue historique et sociologique. Ce travail visait à identifier des familles de pensée et à situer la revue, dont la position s'est toujours manifestée avec prudence et diplomatie.

Si la volonté de s'intéresser au champ de la revue et aux discours tenus sur l'enseignement de la civilisation a pu entraîner une tension, dans la recherche, entre ce qui appartenait au domaine civilisationnel et ce qui relevait de la vie de la revue, l'étude entreprise a permis de constater la constitution et la progression d'une réflexion didactique qui a cherché constamment à s'affiner et où la linguistique, du moins pour la période étudiée a joué un rôle fondamental. L'enseignement de la civilisation, est devenu dans les travaux et articles des méthodologues du BELC un terrain d'application supplémentaire pour la linguistique, ce qui a conduit à un changement en profondeur de la façon dont on envisageait cet enseignement sous l'influence des modifications qui affectaient les représentations de la langue et parallèlement à celles-ci. En dépit des précautions oratoires et des discours conciliateurs, a dominé la volonté de rompre avec un héritage humaniste jugé dépassé et dont le maintien était même jugé dangereux. D'une façon générale, à un objectif ancien - faire percevoir des valeurs, répandre une certaine image de la France - s'est substitué un objectif nouveau qui était de démythifier les valeurs anciennes sur lesquelles la France avait fondé sa présence à l'étranger. Ce qui explique que la réflexion didactique sur l'enseignement de la civilisation se soit construite autour de l'interrogation sur les contenus, sur la recherche des documents et des supports les plus appropriés à cet objectif de démythification, accompagnés du traitement adéquat de ces documents. Inscrite dans une politique globale de renouveau de l'action culturelle dans les années 60 et dont les grands principes avaient été tracés par le Général de Gaulle (renoncement aux colonies, substitution de la contrainte par une politique préférentielle de coopération, restauration du prestige de la France sur la scène internationale, réajustement des ambitions, respect des cultures), la revue fera siens de tels principes dans la réflexion didactique qu'elle va développer dans ses pages : préoccupation pour le lecteur étranger et appel fréquent à celui-ci, redéfinition de " civilisation " comme synonyme de "culture " au sens anthropologique, démythification de l'image de la France. Lieu de production symbolique, elle a contribué à créer de nouvelles légitimités, à renverser des "schèmes de perception " anciens.

23 La faible représentation du parti des anciens signalait à la fois son anachronisme et son élimination pratiquement déjà consommée. Si l'on reprend la distinction classique utilisée par Raoul Girardet entre progressistes et conservateurs dans son ouvrage L'idée coloniale en France, on peut dire que la revue a permis d'éliminer du champ du F.L.E les conservateurs pour laisser la place aux progressistes, ou que le champ du F.L.E a fait de la revue un espace où cette élimination est devenue à la fois visible et effective. De même que les partisans de l'indépendance des colonies acceptaient de "se soumettre à la 
loi inéluctable de l'évolution des peuples coloniaux et s'efforcent de définir dans certains formes et sous certaines conditions, les fondements d'un ordre nouveau plus conforme aux principes de la justice et de l'équité $»^{13}$, de même l'attitude progressiste, telle qu'elle s'est manifestée dans la revue, résultat de la pression exercée par les changements historiques, ayant fait de nécessité vertu, était prête à construire, dans le domaine du F.L.E et de l'enseignement de la civilisation, un ordre pédagogique et didactique plus conforme au principe de l'égale dignité des cultures, en favorisant l'abandon des anciens mythes et de la culture françaises, ou du moins en rendant impossible et taboue leur manifestation. De ce fait, Le Français dans le Monde, et le titre même de la revue en témoigne, n'a pas été seulement un outil pédagogique; il a contribué également à donner forme à une politique culturelle et à participer au travail de maintien de la présence française dans le monde sur des principes renouvelés. Dans cette entreprise, l'enseignement de la civilisation a joué un rôle fondamental, il a aidé le champ du F.L.E à faire le deuil de la notion d'universalité et de l'ethnocentrisme qui l'accompagnait, et à rejeter un passé dont les conceptions et les valeurs périmées étaient rendues responsables de la défaite et du déclin de la France sur la scène internationale. Sa fonction idéologique a bien été de conjurer l'affaiblissement du « rayonnement » de la France en périmant les représentations anciennes, et c'est cette fonction qui a donné aux nouvelles pratiques pédagogiques proposées par les spécialistes du domaine leur cohérence d'ensemble.

\section{NOTES}

1. On citera en particulier l'ouvrage de Daniel Coste : Aspects d'une politique de diffusion du français langue étrangère depuis 1945, Matériaux pour une histoire, Hatier, Paris, 1984, ainsi que la thèse de Sophie Moirand Une histoire de discours... une analyse des discours de la revue LE FRANÇAIS DANS LE MONDE, 1961-1981, Paris, Hachette, 1988.

2. Louis Porcher La Civilisation, Collection Didactique des langues étrangères, Clé International, 1986.

3. Le sujet est toujours aussi actuel: il suffit de lire dans la presse (Le Monde en particulier) les nombreux articles portant sur la guerre d'Algérie ou les violences policières des années 60 en France.

4. Régine Robin, «L'Analyse du Discours entre la linguistique et les sciences humaines : l'éternel malentendu » dans Langages, $\mathrm{n}^{\circ} 81$, mars 86.

5. Emile Benveniste, Problèmes de linguistique générale, p. 242, Gallimard, Tel, 1996.

6. Nous devons beaucoup ici au travail rigoureux effectué par Sophie Moirand dans sa thèse Une histoire de discours... une analyse des discours de la revue LE FRANÇAIS DANS LE MONDE, 1961-1981, Paris, Hachette, 1988.

7. Dominique Maingueneau, Initiation aux méthodes de l'analyse de discours, p. 121, Hachette Université, 1983.

8. au sens que le sociologue Pierre Bourdieu donne à ce terme.

9. Pierre Bourdieu, Raisons pratiques, p. 45, Les Editions de Minuit, 1994.

10. Pierre Bourdieu, Le sens pratique, p. 88, Les Editions de Minuit, 1980. 
11. Catherine Berbrat-Orecchioni, L'énonciation de la subjectivité dans le langage, p. 18, A. Colin, 1984.

12. H.Mendras, Eléments de sociologie, p. 61, A. Colin, coll U, 1997.

13. Raoul Girardet, L'idée coloniale en France, id, p. 363, Hachette, Pluriel, 1995. 